\title{
Discrete Dynamical Systems: A Brief Survey
}

\author{
Sara Fernandes", Carlos Ramosa，Gyan Bahadur Thapa ${ }^{\mathrm{b}}$ ，Luís Lopes ，Clara Grácio \\ ${ }^{a}$ School of Science and Technology, Universidade de Évora, Research centre CIMA, Portugal \\ ${ }^{\mathbf{b}}$ Pulchowk Campus, Institute of Engineering, Tribhuvan University, Nepal \\ ${ }^{\mathbf{c}}$ Instituto Superior de Engenharia de Lisboa, Research centre CIMA, Portugal \\ Corresponding author: saf@uevora.pt
}

Received: June 27, $2017 \quad$ Revised: Nov 25, $2017 \quad$ Accepted: Jan 5, 2018

\begin{abstract}
Dynamical system is a mathematical formalization for any fixed rule that is described in time dependent fashion. The time can be measured by either of the number systems - integers, real numbers, complex numbers. A discrete dynamical system is a dynamical system whose state evolves over a state space in discrete time steps according to a fixed rule. This brief survey paper is concerned with the part of the work done by José Sousa Ramos [2] and some of his research students. We present the general theory of discrete dynamical systems and present results from applications to geometry, graph theory and synchronization.
\end{abstract}

Keywords: Dynamical systems, iteration, symbolic dynamics, invariants, conductance, synchronization

\section{Introduction}

The study of the so called complex phenomena has become increasingly important for many scientific areas. After profound conceptual transformations, through the 20th century, in physics (relativity, quantum mechanics), in biology and life sciences (Darwin, DNA), the development of computers, an enormous development in the social sciences and an exponential growth of technological diversity, it is clear the current difficulty to understand and deal with these types of phenomena. Its diversity is enormous so is the difficulty of systematization, existing a dispersion of models and approaches.

In part, this dispersion is due to the fact that the topic has been developed more or less simultaneously by different researchers in different specialized sciences. Although the referred researchers generally had mathematical knowledge, there is a lack of abstraction ability to capture the essence and what is common in the complex phenomena, beyond solving particular problems. Therefore, there is a lack of unifying conceptual tools for the formation of a coherent scientific paradigm. There are those who defend the absence of a general theory of complex systems. Our research group assumes the existence of general laws, for now unknown, and seek 
its clarification and formalization. In contrast to the explosive development in the applied areas, the mathematics of nonlinear science and complexity has been developed slowly but constantly, since Poincaré.

The complex systems have the feature of having many interacting parts. The traditional application of analysis will lead to taking, at some point, a limit which in a certain sense is a simplifying step in the description of the system. This was useful for the linear systems or to the use of a purely statistical description (as in statistical physics and quantum mechanics). However, in complex and nonlinear systems this conceptual process leaves the essential aspects of the systems unknown. Completely new approaches are probably necessary to deal with turbulence, equilibrium in biology or economy, intelligence, to clearly enunciate and define measure(s) of the complexity of the systems. It can be accomplished with the careful articulation of known techniques and the development of new in several contexts. In this paper, we present a survey of the work done by Sousa Ramos and some of his students, concerning the following two main topics:

A. Multidisciplinary: Graph theory - conductance [3] and Geometry - isoperimetric inequalities, systoles [4].

B. Networks: Synchronization [6] and Chaotic windows [6].

We do not give exhaustive proofs of the results, but instead we aim to encourage new researchers to embrace this field of mathematics for their own subject.

\section{Iteration}

In this section, we present part of the iteration theory of interval maps in a generic set $X$, developed by several mathematicians, over several years; define topological invariants and appropriate metrics; introduce the theory of symbolic dynamics; establish an interesting and fruitful correspondence between Discrete Dynamical Systems, Markov Chains and Graphs.

Let $X$ be a set (with a structure: topological, measure, metric or other to define later). Let $f$ be a map on $X$ (with its properties: continuity, differentiability or other). The discrete dynamical system $(X, f)$, associated with $f$ in $X$ is defined as $x_{n}=f\left(x_{n-1}\right)=f^{n}\left(x_{0}\right)$, where $x_{0} \in X$, is a given initial iteration value and $f^{n}$ denotes the composition of $f$ with itself $n$ times. In particular, $f^{1}=f$ and $f^{0}$ is the identity map on $X$. For a given $x_{0} \in X$, define its orbit by $f$ as the sequence $x_{0}, x_{1}, x_{2}, x_{3}, \ldots$. If this sequence is periodic, i.e., if there is $n \in N$ such that $f^{n}\left(x_{0}\right)=x_{0}$, we call $x_{0}$ a periodic point with period $n$. If $x_{0}$ is a periodic point with period one, we call it a fixed point.

\subsection{Symbolic Dynamics and Markov Partitions}

Let $X=I \subset \mathbb{R}$ be an interval of real numbers. Consider a map $f: I \rightarrow I$.

Definition. A map $f$ is in the class $M(I)$ if it satisfies the following properties: 
(P0) $\operatorname{Im}(f)=I$.

(P1) There is a partition $C=\left\{I_{1}, \ldots, I_{n}\right\}$ of open intervals with $I_{i} \cap I_{j}=\varnothing$ for $i \neq j, I=$ $\bigcup_{j=1, \ldots, n} I_{j}$.

(P2) For every $j=1, \ldots, n$ the set $f\left(I_{j}\right)$ is a non-empty union of intervals from $C$.

(P3) $f_{\left.\right|_{I_{j}}} \in C^{1}\left(I_{j}\right)$, is monotone and $\left|f^{\prime}(x)\right|>b>1$, for every $x \in I_{j}, j=1, \ldots, n$, and some $b$.

(P4) For every interval $I_{j}$ with $j=1, \ldots, n$ there is a natural number $q$ such that $\operatorname{dom}(f) \subset$ $\overline{f^{q}\left(I_{j}\right)}$.

We call every partition verifying the properties of the previous definition a $f$-partition. The minimal $f$-partition is called Markov partition of $f$ and it is denoted by $C_{f}$. We deal here mainly with the minimal partition $C_{f}$, and in this case, $n$ the cardinality of the Markov partition, is a fixed parameter of $f$. The set $I \backslash \bigcup_{j=1, \ldots, n} I_{j}$ is the set of singular points and from the above definition, it is a finite set.

A map $f \in M(I)$ together with the minimal partition $C_{f}=\left\{I_{1}, \ldots, I_{n}\right\}$ uniquely determines:

(a) The $f$-invariant set, $\Omega_{f}:=\left\{x \in I: f^{k}(x)\right.$ is in $U_{j=1, \ldots, n} I_{j}$ for all $\left.k=0,1, \ldots\right\}$.

(b) A transition matrix, $A_{f}=\left(a_{i j}\right)_{i, j=1, . ., n}$, defined by:

$$
a_{i j}=\left\{\begin{array}{cc}
1 & \text { if } f\left(I_{i}\right) \supset I_{j} \\
0 & \text { otherwise }
\end{array} .\right.
$$

Thus, $\Omega_{f}$ is the set of points that remain in $\bigcup_{j=1, \ldots, n} I_{j}$ under iteration of $f$. Note that if $f \in M(I)$, then $f^{k} \in M(I)$ for every $k \in \mathbb{N}$.

Let $[n]:=\{1,2, \ldots, n\}$ be the alphabet indexing the elements of $C_{f}$. The itinerary map $i t_{f}$ : $\Omega_{f} \rightarrow[n]^{\mathbb{N}}$ is defined by $i t_{f}(x):=\left(i_{j}\right)_{j} \in \mathbb{N}$ where $i_{j}$ is the index in $[n]$ such that $x_{j} \in I_{j}$.

Let $\Sigma_{f}$ be the subspace of $[n]^{\mathbb{N}}$ given by $i t_{f}\left(\Omega_{f}\right)$, which is invariant under the shift map $\sigma:[n]^{\mathbb{N}} \rightarrow[n]^{\mathbb{N}}$ defined as $\sigma\left(i_{1} i_{2} i_{3} \ldots\right)=\left(i_{2} i_{3} \ldots\right)$

We will use just $\sigma$ instead of $\sigma_{\left.\right|_{f}}$. Naturally, $\Sigma_{f}=\left\{i_{1} i_{2} i_{3} \ldots: a_{i_{k} i_{k+1}}=1, k \in \mathbb{N}\right\}$ and $i t_{f} \circ f=\sigma \circ i t_{f}$. The pair $\left(\Sigma_{f}, \sigma\right)$ is a subshift of finite type, characterized by the matrix $A_{f}$. Since $f$ is expansive (due to $\mathrm{P} 3$ ), we know that $\left(\Omega_{f}, f\right)$ is topologically conjugated to $\left(\Sigma_{f}, \sigma\right)$ via the itinerary map $i t_{f}: \Omega_{f} \rightarrow \Sigma_{\mathrm{f}}$. In fact, the map $i t_{f}$ is surjective by construction of $\Sigma_{f}$. Is injective, because the inverse branches $\left(f^{-1}\right)_{\mathrm{I}_{\mathrm{j}}}$ are monotone and contractive in every point of its domain $f\left(I_{j}\right)$. Now, given a sequence $\left(i_{j}\right)_{j \in \mathbb{N}} \in \Sigma_{f}$ the sequence of intervals $I_{i_{1} i_{2} \ldots i_{k}}$, with $k \geq 1$, satisfies

$$
I_{i_{1} i_{2} \ldots i_{k+1}} \subset I_{i_{1} i_{2} \ldots i_{k}} \text { and } \lim _{k \rightarrow \infty}\left|I_{i_{1} i_{2} \ldots i_{k}}\right|=0 .
$$

Therefore, the boundary points of $I_{i_{1}} i_{2} \ldots i_{k}$ converge to a point, which is the unique pre-image of 
$i t_{f}$, i.e., $i t_{f}$ is invertible. The continuity results from the choice of the discrete topology in both spaces, $\Omega_{f}$ and $\Sigma_{\mathrm{f}}$.

A sequence in $[n]^{\mathbb{N}}$ is called admissible, with respect to $f$ if it occurs as an itinerary of some point $x$ in $\Omega_{f}$, i.e., if it belongs to $\Sigma_{f}$. A periodic sequence in $\Sigma_{f}$ is an admissible periodic sequence $\begin{array}{llllllllll}i_{1} & i_{2} & \ldots & i_{k} i_{1} i_{2} & \ldots & i_{k} i_{1} & \ldots & =\end{array}=\left(\begin{array}{lllll}i_{1} & i_{2} & \ldots & i_{k}\end{array}\right)^{\infty}$. To learn more on symbolic dynamics see [5].

\subsection{Sharkovsky Theorem}

A continuous map of the interval is conditioned by the Sharkovsky Theorem regarding the periods. As we will see below this result is very strong and restrictive. It forces, for example, that a map with a periodic point other than the fixed point, to have a period 2.

Theorem (Sharkovsky): Consider the following order of the natural numbers:

$$
\begin{aligned}
3 \triangleright 5 \triangleright 7 \triangleright \cdots \triangleright & (2 n+1) \cdot 2^{0} \triangleright \cdots \triangleright 2^{1} \cdot 3 \triangleright 2^{1} \cdot 5 \triangleright 2^{1} \cdot 7 \triangleright \cdots \triangleright(2 n+1) \cdot 2^{1} \triangleright \cdots \\
& \triangleright 2^{2} \cdot 3 \triangleright 2^{2} \cdot 5 \triangleright 2^{2} \cdot 7 \triangleright \cdots \triangleright(2 n+1) \cdot 2^{2} \triangleright \cdots \ldots \triangleright 2^{n} \cdot 3 \triangleright 2^{n} \cdot 5 \\
& \triangleright 2^{n} \cdot 7 \triangleright \cdots \triangleright(2 n+1) \cdot 2^{n} \triangleright \cdots \triangleright 2^{n} \triangleright \cdots \triangleright 2^{3} \triangleright 2^{2} \triangleright 2 \triangleright 1 .
\end{aligned}
$$

Suppose $f: \mathbb{R} \rightarrow \mathbb{R}$ continuous. Suppose f has a periodic point of period $k$. If $k \triangleright p$ in the above ordering, then falso has a periodic point of period $p$.

\subsection{Iteration, Markov Chains and Graphs}

Associated to each map $f \in M(I)$ with transition matrix $A_{f}=\left(a_{i j}\right)$, we define a directed graph (digraph) $G_{f}$ with vertex set $V_{f}=C_{f}$ and edge set $E_{f}=\left(I_{i}, I_{j}\right) \in C_{f}^{2}: a_{i j}=1$. From this definition, the adjacency matrix of $G_{f}$ is precisely the transition matrix $A_{f}$. We, therefore, obtain a natural correspondence between the orbits of $f$ and the paths on $G_{f}$. In this sense, the cycles of $\mathrm{f}$ correspond to closed paths on $G_{f}$. Later in this text, we provide a concrete example of the path

$$
(X, f) \rightarrow A_{f} \rightarrow\left(\Sigma_{f}, \sigma\right) \rightarrow G_{f}
$$

\subsection{Invariants}

Symbolic dynamics is an important tool to study piecewise monotone interval maps. From the periodic orbits of the images of the critical (or discontinuity) points of a piecewise monotone map $f$ on an interval is possible to calculate important invariants that characterize the whole dynamics such as the topological entropy, $h_{\text {top }}(f)$ (see [7]). In this section, we are particularly interested in the $m$-modal class of interval maps. The $m$-modal class is formed by continuous maps on the interval with $m$ critical points. That means that we have piecewise monotone functions with $(m+1)$ laps of monotonicity.

By a result of Parry, see [8], for each unimodal map $f$ there is only one piecewise linear map $F$ (by semi-conjugation) defined on the interval $[0,1]$ with slope $\pm s$, so that $h_{\text {top }}(f)=h_{\text {top }}(F)=$ $\log \mathrm{s}$. The topological entropy is a complete invariant for the piecewise linear unimodal family. 
In the case of $f \in M(I)$, this parameter s, called growth number, can be calculated as the greater eigenvalue of the transition matrix. As a measure of chaoticity of the one-dimensional discrete dynamical system of the iterates of $f$, we use the Lyapunov exponent, which is defined as

$$
\mu=\lim _{T \rightarrow \infty} \frac{1}{T} \sum_{n=0}^{T-1} \ln \left|f^{\prime}\left(x_{n}\right)\right|
$$

In the case of continuous m-modal maps with constant slope, it is simply the topological entropy. The research for new invariants has been one of the main objectives of our group. Algebraically, besides $s$, there is the parameter $r$, introduced by Sousa Ramos in [1] which has a similar role as the slope $s$ in unimodal maps but applied to the family of bimodal maps. The use of the algebraic parameters $s$ and $r$ allow to define a complete family of bimodal maps concerning the possible dynamics.

\section{Multidisciplinary}

\subsection{Graph Theory: Conductance}

In this section, we present two other invariants (although not complete) in these m-modal families of maps: the mixing rate and the conductance.

The models we have in mind are provided by the family of unimodal (tent) maps, $T_{S}: I \rightarrow I$, depending on one parameter $s \in(1,2]$ and defined by

$$
T_{s}(x)=\left\{\begin{array}{cc}
s x & \text { if } 0 \leq x<1 / 2 \\
-s x+s & \text { if } 1 / 2 \leq x \leq 1
\end{array}\right.
$$

and by the family of bimodal maps, $T_{s, e}: I \rightarrow I$, depending on two parameters, $s \in(1,3]$ and $e \in[s-1,2]$ defined by

$$
T_{s, e}(x)=\left\{\begin{array}{cc}
s x & \text { if } 0 \leq x<c_{1} \\
-s x+e & \text { if } c_{1} \leq x<c_{2} \\
s x+1-s & \text { if } c_{2} \leq x<1
\end{array}\right.
$$

where $c_{1}=e /(2 s)$ and $c_{2}=(e+s-1) /(2 s)$ are the critical (turning) points.

The choice of these piecewise linear families is just an example and is not important. Actually, they are all topologically conjugated (see [7]) to families of the same modality and so, its qualitative behaviour is the same. If each trajectory of the critical points in these maps is periodic there is a Markov partition induced by the points where the trajectories goes through. First, we introduce the address of $x \in[0,1]$ for unimodal maps by

$$
\operatorname{ad}(x)=\left\{\begin{array}{l}
L \quad \text { if } 0 \leq x<c \\
C \quad \text { if } x=c \\
R \quad \text { if } c<x \leq 1
\end{array}\right.
$$


where $c$ is the critical point. In the considered unimodal family, this is always $c=1 / 2$, and the address of $x \in[0,1]$ for bimodal maps by

$$
T_{s, e}(x)=\left\{\begin{array}{cc}
L & \text { if } 0 \leq x<c_{1} \\
A & \text { if } x=c_{1} \\
M & \text { if } c_{1}<x<c_{2} \\
B & \text { if } x=c_{2} \\
R & \text { if } c_{2}<x \leq 1
\end{array}\right.
$$

where $c_{1}$ and $c_{2}$ are the critical points.

Secondly, we consider the periodic symbolic itineraries of the critical point, $\left(C P_{1} P_{2} \ldots P_{k}\right)^{\infty}$ for unimodal maps or the pair of symbolic itineraries of both critical points $\left(\left(A P_{1} P_{2} \ldots P_{k}\right)^{\infty},\left(B Q_{1} Q_{2} \ldots Q_{m}\right)^{\infty}\right)$.

These itineraries give us the points of the interval that will define the Markov partition as stated above. Thus, considering the points of the interval $I$ where the trajectory or trajectories goes by we obtain a partition $C$ of $I$ which satisfies the properties of Definition 3.1. These points are codified

$$
\left(C P_{1} P_{2} \ldots P_{k}\right)^{\infty},\left(P_{1} P_{2} \ldots P_{k} C\right)^{\infty},\left(P_{2} \ldots P_{k} C P_{1}\right)^{\infty}, \ldots,\left(P_{k} C P_{1} P_{2} \ldots P_{k-1}\right)^{\infty},
$$

in the unimodal case and for the bimodal case the situation is similar but we have $k+m$ points. In fact, it can be at most $k+m$ because some points of the itineraries can coincide.

We present a simple example of a unimodal function of the interval with a 4-periodic trajectory of the critical point.

Example: Identify the trajectories of the points $x_{1}, x_{2}, \ldots, x_{4}$, in the 4-periodic trajectory of the critical point with its symbolic itinerary.

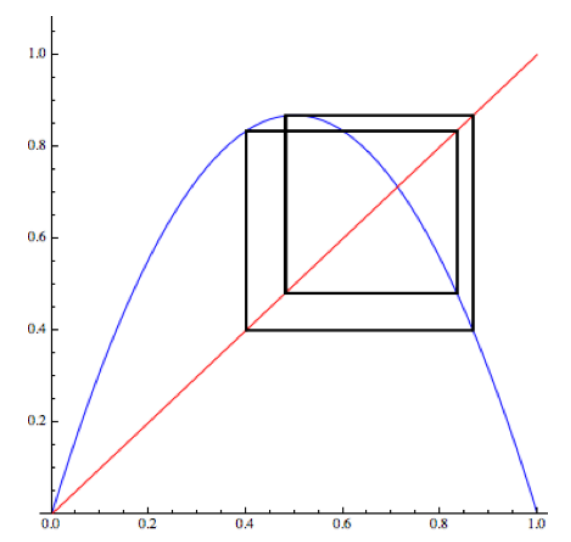

$$
\begin{aligned}
& (\mathrm{CRLR})^{\infty} \rightarrow(\mathrm{RLRC})^{\infty} \rightarrow(\mathrm{LRCR})^{\infty} \rightarrow(\mathrm{RCRL})^{\infty} \\
& \mathrm{x}_{1} \rightarrow \mathrm{x}_{2} \rightarrow \mathrm{x}_{3} \rightarrow \mathrm{x}_{4} \\
& \mathrm{x}_{3}<\mathrm{x}_{1}<\mathrm{x}_{4}<\mathrm{x}_{2} \\
& \mathrm{I}_{1}=\left[\mathrm{x}_{3}, \mathrm{x}_{1}\right], \quad \mathrm{I}_{2}=\left[\mathrm{x}_{1}, \mathrm{x}_{4}\right], \quad \mathrm{I}_{3}=\left[\mathrm{x}_{4}, \mathrm{x}_{2}\right] \\
& \mathrm{f}\left(\mathrm{I}_{1}\right)=\mathrm{I}_{3} \quad \mathrm{f}\left(\mathrm{I}_{2}\right)=\mathrm{I}_{2} \cup \mathrm{I}_{3} \quad \mathrm{f}\left(\mathrm{I}_{3}\right)=\mathrm{I}_{1}
\end{aligned}
$$

The partition gives rise to the transition matrix $A_{f}$, and to the graph $G_{f}$, which adjacency matrix is $A_{f}$. 


$$
A_{f}=\left(\begin{array}{lll}
0 & 0 & 1 \\
0 & 1 & 1 \\
1 & 0 & 0
\end{array}\right)
$$

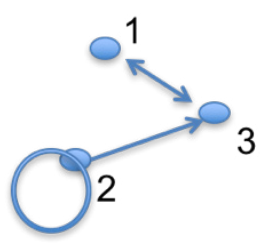

This example has more importance than we can see in a first sight. In fact, there is a one-to-one correspondence between the periodic symbolic trajectories of the critical point and the oneparameter family of unimodal maps, considered above. More precisely, we use the trajectory of the image of the critical point, $f(c)$, the maximum of the map and we call it kneading sequence [7]. To this trajectory will correspond a maximal symbolic itinerary. These symbolic sequences can be ordered in an infinite binary tree, which projection in the real line gives the growth number of the corresponding map. The order in the itineraries depends on the number of symbols corresponding to the decreasing laps of the map (Fig. 1).

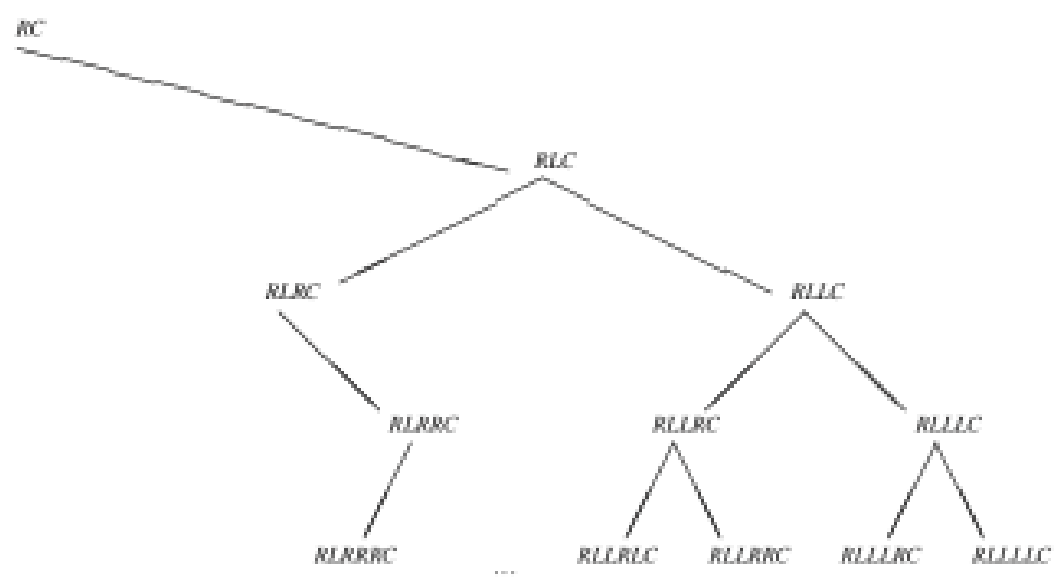

Fig. 1: Ordered tree of kneading sequences

Similar results are stated for m-modal maps with the increase of complexity resulting from the existence of $m$ kneading sequences.

From the adjacency matrix $A_{f}$, we can define an ergodic Markov chain, adding weights to the edges of the graph $G_{f}$. These weights can be seen as measures of probabilities to move from one vertex (state) to another. We can do it, from the adjacency matrix $A_{f}$, in several ways, depending on the point of view we want to emphasise. The simplest one is devising the entries of each row of the matrix $A_{f}$ by the sum of (positive) entries in this same row, obtaining the matrix $P$, with the following important properties, which characterize a stochastic matrix:

P1. $\sum_{j} p_{i j}=1$ for all $i$ 
P2. $p_{i j} \geq 0$ for all $i j$

P3. there is a vector $\pi$ such that $\pi P=\pi$

We say that the stochastic matrix $P$ is ergodic, if we add the property:

P4. there is a $k$ such that, all entries of the matrix $P^{k}$ are strictly positive, i.e., it is possible to go from every state to every state in at most $k$ steps

If the matrix $A_{f}$ is aperiodic, then there exists a different weighted stochastic matrix $P$ associated with the measure of maximal entropy, the Parry measure, defined as follow (see [9]). Let $u$ and $v$ be the normalized left and right (respectively) Perron eigenvectors of $A_{f}$, so that $\sum u_{i} v_{i}=1$. Let $\lambda$ be the Perron eigenvalue of the matrix $A_{f}$. Then the matrix $P=\left(p_{i j}\right)_{i, j=1, \ldots, n}$, defined by

$$
p_{i j}=\frac{a_{i j}}{\lambda} \frac{v_{j}}{v_{i}}
$$

satisfy $\sum_{j=1}^{n} p_{i j}=1$. Moreover, the vector $p=\left(p_{i}\right)_{i=1, \ldots, n}$, with $p_{i}=u_{i} v_{i}$ is the left Perron eigenvector of $P$ with eigenvalue 1 and corresponds to the unique invariant measure of maximal entropy.

We will see in the next section the consequences of a different choice of weights. A Markov chain represented by an ergodic stochastic matrix $P$ converges to a stationary or equilibrium distribution. It is possible to obtain an explicit bound for this convergence based on the eigenvalues of $A_{f}$. We have the following result.

Theorem: Let $f \in M(I)$ be a piecewise linear map with constant slope $s>1$ in every interval of the Markov partition and let $A_{f}$ be the transition matrix. Let $\lambda_{1}$ and $\lambda_{2}$ be the first and second eigenvalue (in magnitude), of the matrix $A_{f}$. Then we have exponential fast convergence, with rate at most $\lambda_{2} / \lambda_{1}$, if $\lambda_{2}>1$ and if $\lambda_{2}<1$, we have convergence rate at most $1 / \lambda_{1}$.

In Figure 2, we can see the behaviour of three quantities related to this convergence: the conductance, $\Phi$, the second eigenvalue of the transition matrix, $\lambda_{2}$, and the mixing time, mix. The conductance of the discrete dynamical system $(I, f)$ is the measure of fluidity in the associated weighted graph, $G_{f}$, where the weighted edges, $e_{i j}$, are the entries $p_{i j}$, of the matrix $P$. The definition follows.

Definition: Consider the discrete dynamical system $(I, f)$, with $f \in M(I)$ and the associated ergodic stochastic matrix $P$. Consider the vector $\pi$, the equilibrium. Define the conductance of the system as

$$
\Phi=\min _{\varnothing \neq U \subset V} \frac{\sum_{i \in U, j \in \bar{U}} \pi_{i} P_{i j}}{\min \left\{\sum_{i \in U} \pi_{i}, \sum_{i \in \bar{U}} \pi_{i}\right\}}
$$

In probabilistic terms, this ratio measures the ability of the system in equilibrium to leave small 
parts of the state space, conditioned by belonging to them. It allowed differentiating systems from the point of view of the fluidity of the system. In fact, this definition captures the existence of small parts from where is difficult to escape, and so, the convergence to equilibrium is slower. If we fix a small distance $\varepsilon>0$ and count the number of iterations to get $\varepsilon$-close to the equilibrium, given that we choose a random initial condition, we obtain the mixing time (the figure in the middle). In the Figure 2, we can see the expected connexions: smaller conductance implies greater mixing time and greater $\lambda_{2}$. The plot in Fig. 2 includes the data of all unimodal maps in the family (1) with the period of the kneading sequence smaller than 8 . The x-axes of all graphs, contain the topological entropy of the corresponding system.

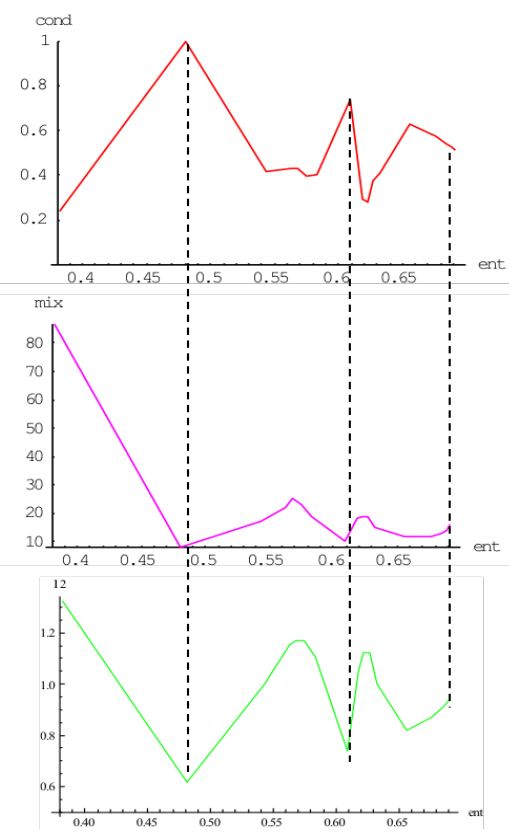

Fig. 2: Extrema in conductance, mixing rate and mixing time in unimodal maps

\subsection{Geometry: Isoperimetric Inequalities, Systoles}

One of the important topological characteristics of a discrete dynamical system is the set of periodic orbits. The growth number of a map is the growth rate of the number of distinct periodic orbits with a given length, and the logarithm of the growth number gives the topological entropy of the map as stated in the Introduction. As we saw, we can analyse the orbits of a given map $f \in M(I)$ using symbolic infinite sequences. In the case the orbits are periodic the corresponding symbolic sequences are also periodic, in the sense that there is an infinitely repeated block. The corresponding a graph $G_{f}$ allows the combinatorial description of the orbits of $f$.

Given $f \in M(I)$ and the underlying graph, $G_{f}$, there is a one-to-one correspondence between the periodic orbits of $f$ and the cycles (closed paths) on the graph $G_{f}$. We call these cycles, the cycles of $f$. A cycle of period $k$ in the graph will be denoted by $\gamma=\left(i_{1} i_{2} \ldots i_{k}\right)^{\infty}$ if it uses the vertices $\mathrm{i}_{1} \mathrm{i}_{2} \ldots \mathrm{i}_{k}$. Note that such a cycle exists in $G_{f}$ if and only if $\mathrm{a}_{\mathrm{i}_{\mathrm{j}} \mathrm{i}_{\mathrm{j}+1}} \neq 0$ for all $j=1, \ldots, k-1$ and 
$\mathrm{a}_{\mathrm{i}_{\mathrm{k}} \mathrm{i}_{1}} \neq 0$. We say that the edges $\left(\mathrm{i}_{p}, \mathrm{i}_{\mathrm{p}+1}\right)$, for $p=1, \ldots, k-1$, and $\square\left(i_{k}, i_{1}\right)$, belong to the cycle $\gamma$. A loop is a cycle with just one vertex $\left(i_{1}\right)^{\infty}$.

Now, we introduce the metrical notions, which are necessary to define the systole. Since the set of edges of $G_{f}, E_{f}$, is finite, to define a measure $v$ on $E_{f}$, it suffices to define $v_{i j}:=v((i, j))$ for each $(i, j) \in E_{f}$.

Definition. Let $f \in M(I)$. We denote by $C y c(f)$ the set of nonloop cycles of $f$.

Definition. Let $f \in M(I)$. Let $v$ be a measure on $E_{f}$. Let $\gamma=\left(\mathrm{i}_{1} \mathrm{i}_{2} \ldots \mathrm{i}_{k}\right)^{\infty} \in \operatorname{Cyc}(S)$. We define the length of $\gamma, l_{v}(\gamma)$, with respect to $v$, by the sum of the measures of each edge in $\gamma$,

$$
l_{v}(\gamma)=\sum_{j=1}^{k} v_{i_{j} i_{j+1}}
$$

considering in this case that $i_{k+1}=i_{1}$.

Definition. Let $f \in M(I)$. The systole of $f$, with respect to a given measure $v$ on $E_{f}$, denoted by $\operatorname{sys}_{v}(f)$, is the smallest length of the cycles of $f$, which are not loops in $G_{f}$. That is,

$$
\operatorname{sys}_{v}(f)=\min \left\{l_{v}(\gamma): \gamma \in C y c(f)\right\} .
$$

If we consider the counting measure, $\delta$, that is, the measure that assigns 1 to each edge of the graph, the length of a cycle is the number of edges (or vertices) that the cycle uses. In this context, the systole will be a natural number, $\operatorname{sys}_{\delta}(f)$.

Let $\mu$ denote the measure on $E_{f}$ arising from the stochastic matrix P defined in (3), as follows

$$
\mu_{i j}=\mu((i j)):=-\log p_{i j}
$$

In this case, the length of a cycle is given by

$$
l_{\mu}(\gamma)=-\sum_{(i, j) \in \gamma} \log p_{i j}=-\log \prod_{(i, j) \in \gamma} p_{i j} .
$$

The probabilistic interpretation of $p_{i j}$ is in agreement with the interpretation of $\mu_{i j}$ as a distance between the vertices $i$ and $j$. It allows us, in probabilistic terms, to distinguish the ability of the system to pass from a state $i$ to a state $j$ in an interval of time. If $p_{i j}$ is large, the distance is short, if $p_{i j}$ is small, the distance is long. If $p_{i j}$ is null, we can say that the distance is infinite.

In Geometry, the systole corresponds to the geodesic of smallest length and corresponds, in weighted graphs, to the cycle which maximize the product $\prod_{(i, j) \in \gamma} p_{i j}$. The fixed point is discarded since it is analogous to the contractible loop in geometry. Therefore, the smallest nontrivial cycle corresponds to the cycle composed of two vertices which is actually a period 2 orbit (if exists).

Assuming the analogy between the geodesics and the cycles in the graph, it is natural to define 
the length spectrum associated with a map $f \in M(I)$, with respect to a measure on $E_{f}$.

Definition: Let $f \in M(I)$. The length spectrum of $f$, with respect to the measure $v$, is the set of lengths of the cycles of $f$. It is denoted by

$$
\operatorname{spec}_{v}(f)=\left\{l_{v}(\gamma): \gamma \in \operatorname{Cyc}(f)\right\} .
$$

Concerning the iterates of a map in the interval, we can ask if the systole is always obtained from the period 2. There are examples arising from discontinuous maps which have no period 2 and we may ask if the systole is always obtained from the minimal period orbit, that is, from the systole associated to the counting measure $\operatorname{sys}_{\delta}(f)$. There is a positive answer, in fact we have the following result.

Proposition: The length of a cycle $\gamma$ of period $k$, with respect to the measure $\mu$ is equal to $l_{\mu}(\gamma)=$ $k \cdot h_{\text {top }}$, where $h_{\text {top }}$ is the topological entropy of the system.

With respect to $\mu$ we have three immediate corollaries.

Corollary: The length of different cycles with the same period are equal.

Corollary: If there exists a period 2, then the length of the corresponding cycle will be the systole. Generally, the systole with respect to $\mu$ will be the length of the shortest non-loop cycle

$$
\operatorname{sys}_{\mu}(f)=\operatorname{sys}_{\delta}(f) \cdot h_{t o p} .
$$

Corollary: With respect to $\mu$ the lengths of the cycles are integer multiples of $h_{\text {top }}$

$$
h_{\text {top }}, 2 h_{\text {top }}, 3 h_{\text {top }}, \ldots, k h_{\text {top }} \text {. }
$$

Proposition: Let $f \in M(I)$. Then, for all positive integers $n$,

$$
\operatorname{sys}_{\mu}\left(f^{n}\right)=\frac{n}{\operatorname{gcd}\left(n, \operatorname{sys}_{\delta}(f)\right)} \operatorname{sys}_{\mu}(f) .
$$

If $f$ is continuous, having a minimal period (discarding the fixed point) then it will have a period two, that is, if $f$ is continuous then sys $f(f)=2$. From the previous Corollaries we conclude that, regarding continuous $m$-modal maps of the interval, the systole does not give much additional information, compared with the topological entropy of the system.

However, in the case of discontinuous functions, the importance of the systole, regarding topological characterization will be justified. A first natural question is if the systole of a given map $f \in M(I)$ depends on the number of discontinuity points of $f$.

Next, a natural question is to ask if there are interval maps with nontrivial minimal period cycle (other than 2). If there is such a map it must be discontinuous. In [4] we show that a map with one discontinuity point can have any minimal period, and therefore we can produce a wide range 
of different systoles for the family of one discontinuity point Markov maps.

To do this we have considered the family $S_{a b c}$, with $1<a, b<2$ and $0<c<1$.

$$
S_{a b c}=\left\{\begin{array}{lll}
a(x-c)+1 & \text { if } & 0 \leq x<c \\
b(x-c) & \text { if } & c \leq x \leq 1
\end{array}\right.
$$

We can see an example of this map in Fig. 3 where are plotted the map $S_{a b c}$, the diagonal line and the trajectories of the remarkable points $0, c$ and $l$.

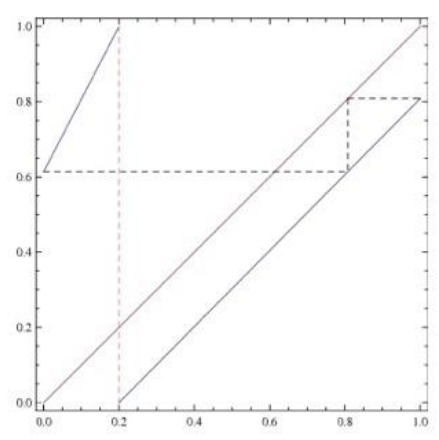

Fig. 3: Map of the family $S_{\text {abc }}$

In the proof of the next theorem, we build a subfamily, with the appropriate restriction to the parameters $a, b, c$, which may have any prescribed minimal period. That is, given a positive integer n, we can choose the parameters $a, b, c$ in order to $S_{a b c}$ has $n$ as its minimal period. Our method is to choose the parameters $a, b$, and $c$ in such a way that all the initial values in the left interval will attain the right interval in one step.

Theorem. Given $n \in \mathbb{N}$, there is a Markov map $f \in M(I)$, which has one discontinuity point, with minimal period $n$.

The proof of this theorem is based on algebraic manipulations on the parameters and on the matrix $A_{f}$. We can see an Example in Fig. 4.
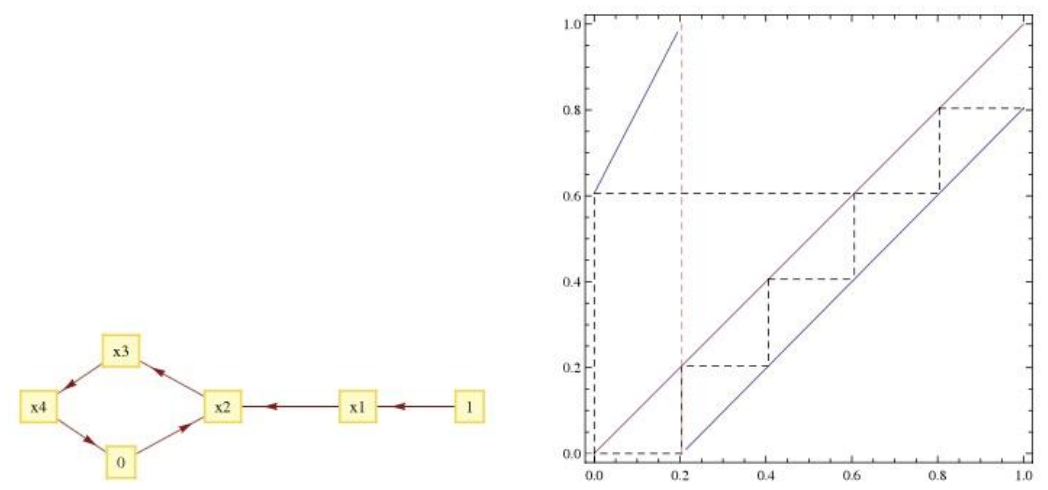

Fig. 4: Trajectory of 0 and 1 , on the left, and the map $S_{a b c}$, on the right, for the case without period 3. 
In [4] we analysed interval maps with one discontinuity point, regarding systoles associated with the counting measure (minimal periods) and the Parry measure (which relates the systole with the topological entropy). This discontinuity point is necessary in order to have a non-trivial period (different from period two). One might ask what are the differences for interval maps with more than one discontinuity point. It is known that the maximal topological entropy we may obtain in an interval map with $\mathrm{n}$ discontinuity points is $\log (n+1)$, however in this case the systole will be the trivial one (there is a fixed point). On the other hand, with one-discontinuity point interval maps it is possible to produce examples with an arbitrary minimal period. However, if we fix the topological entropy, lower than $\log (n+1)$, it is not clear that we may build examples with different minimal periods, since the value of $\alpha$ is strongly related with $\beta$. Therefore, it is necessary to introduce new discontinuity points in order to be able to fix the topological entropy and to vary freely the minimal period.

\section{Networks}

\subsection{Synchronization}

In this section, we introduce a new point of view concerning discrete dynamical systems. What happens if we couple together two or more systems? Do they synchronize? In what conditions? What can we expect of the invariants, as the Lyapunov exponent? What happens with the chaos? In several works, we obtained the conditions that a general coupling needs to satisfy in order to admit a complete synchronization or a delayed synchronization (known in the literature as lag synchronization). We consider a coupling of two discrete one-dimensional chaotic dynamical systems, $x_{1}$ and $x_{2}$, with the same dynamics defined by the map $f$.

$$
\left\{\begin{array}{l}
x_{1}(t+1)=f\left(x_{1}(t)\right)+c \cdot\left[g_{12}\left(x_{2}(t)\right)-g_{11}\left(x_{1}(t)\right)\right] \\
x_{2}(t+1)=f\left(x_{2}(t)\right)+c \cdot\left[g_{21}\left(x_{1}(t)\right)-g_{22}\left(x_{2}(t)\right)\right]
\end{array}\right.
$$

where time parameter $t$ is integer, $c \in \mathbb{R}$ is the coupling strength and $g_{i j}$ are real functions for $i$, $j=1,2$.

The most common coupled system in the literature is the Symmetric Linear Coupled System (SL), i.e., the system corresponding to $g_{i j}=f$, for $i, j=1,2$.

$$
\left\{\begin{array}{l}
x_{1}(t+1)=f\left(x_{1}(t)\right)+c \cdot\left[f\left(x_{2}(t)\right)-f\left(x_{1}(t)\right)\right] \\
x_{2}(t+1)=f\left(x_{2}(t)\right)+c \cdot\left[f\left(x_{1}(t)\right)-f\left(x_{2}(t)\right)\right]
\end{array}\right.
$$

For the system (4), we consider two types of synchronization - complete synchronization and delayed synchronization - as established in the following definitions.

Definition: We say that the coupled system (4) admits a completely synchronized solution if there is a function $s(t)$ such that $\left(x_{1}(t), x_{2}(t)\right)=(s(t), s(t))$ is a solution of $(4)$. 
Definition: We say that the coupled system (4) admits a synchronized-with-delay solution if there is a non- constant function $\mathrm{s}(\mathrm{t})$ such that $\left(x_{1}(t), x_{2}(t)\right)=(s(t), s(t-1))$ is a solution of (4).

The ability of the system (4) to admit a synchronized solution is related to conditions that the functions $g_{i j}$ must satisfy.

Proposition: The coupled system (4) admits a chaotic completely synchronized solution $\left(x_{1}(t), x_{2}(t)\right)=(s(t), s(t))$, if and only if $g_{11}-g_{12}+g_{21}-g_{22}=0$ and $\mu_{1}>0$ where

$$
\mu_{1}=\lim _{N \rightarrow \infty} \frac{\sum_{t=0}^{N} \ln \left|f^{\prime}+c\left(g_{12}^{\prime}-g_{11}^{\prime}\right)\right|_{s(t)}}{N}
$$

The Proposition above ensures that the SLCS admits a chaotic completely synchronized solution. It also ensures it for another useful coupled system, the Commanded Linear Coupled System (CL), i.e., the coupling corresponding to $g_{11}=g_{12}=0 \wedge g_{22}=g_{21}=f$.

Proposition Consider the coupled system (4). Then

1. It admits a chaotic synchronized-with-delay solution if and only if $g_{12}=g_{11} \circ f \wedge g_{22}=$ $g_{21} \circ f$

2. It admits a period-2 synchronized-with-delay solution if and only if $g_{21}=g_{12} \wedge g_{22}=g_{11}$ where, by period-2 solution we mean for all $t,\left(x_{1}(t-1), x_{2}(t-1)\right)=\left(x_{1}(t+1), x_{2}(t+\right.$ 1)).

The SL admits a period-2 synchronized-with-delay solution, but it does not admit a chaotic one. The CL does not admit any synchronized-with-delay solution.

Taking in account the Proposition above, we consider two variations of these coupled systems that will admit synchronized-with-delay solutions:

- The Commanded Coupled System with Delay (CD):

$$
\left\{\begin{array}{l}
x_{1}(t+1)=f\left(x_{1}(t)\right) \\
x_{2}(t+1)=f\left(x_{2}(t)\right)+c \cdot\left[x_{1}(t)-f\left(x_{2}(t)\right)\right]
\end{array}\right.
$$

- The Symmetric Coupled System with Delay (SD):

$$
\left\{\begin{array}{l}
x_{1}(t+1)=f\left(x_{1}(t)\right)+c \cdot\left[x_{2}(t)-f\left(x_{1}(t)\right)\right] \\
x_{2}(t+1)=f\left(x_{2}(t)\right)+c \cdot\left[x_{1}(t)-f\left(x_{2}(t)\right)\right]
\end{array}\right.
$$

The CD admits a chaotic synchronized-with-delay solution but does not admit a period-2 one. The SD behaves the opposite way: it admits a period-2 synchronized-with-delay solution, but it does not admit a chaotic one. Further, the SD admits a completely synchronized solution, while the $\mathrm{CD}$ does not. 
Even if a coupled system admits a synchronized solution that solution may never appear in numerical simulations because it may be unstable. So, it is important to check if the synchronized solutions are stable. This is done in the next section.

\subsection{Chaotic Windows}

In order to study the stability of the synchronized solutions, we begin by considering the following definitions:

Definition: We say that the coupled system (4) completely synchronizes if the completely synchronized solution $\left(x_{1}(t), x_{2}(t)\right)=(s(t), s(t))$ is an exponentially stable solution of (4). We call "window of complete synchronization" the set of values of the coupling strength $c$ for which the coupled system completely synchronizes. We say that the coupled system (4) synchronizes-with-delay if the synchronized-with-delay solution $\left(x_{1}(t), x_{2}(t)\right)=(s(t), s(t-$ $1)$ ) is an exponentially stable solution of (4). We call "window of delayed synchronization" the set of values of the coupling strength $\mathrm{c}$ for which the coupled system synchronizes-with-delay.

The "window of complete synchronization" for the SL has already been obtained by several authors but very few results were produced for other couplings. We obtained the "window of complete synchronization" for all the couplings that admit a chaotic completely synchronized solution. The results are in the following table.

Table 1: Windows of synchronization

\begin{tabular}{|c||c|c|}
\hline & Window of complete synchronization & $\begin{array}{c}\text { Window of delayed } \\
\text { synchronization }\end{array}$ \\
\hline SL & $\int \frac{1-e^{-\mu_{0}}}{2}, \frac{1+e^{-\mu_{0}}}{2}[$ & empty \\
\hline $\mathrm{CL}$ & ] $1-e^{-\mu_{0}}, 1+e^{-\mu_{0}}[$ & empty \\
\hline $\mathrm{SD}$ & $\left\{c \in \mathbb{R}: \lim _{N \rightarrow \infty} \frac{\sum_{t=0}^{N} \ln \left|(1-c) f^{\prime}-c\right|_{s(t)}}{N}\right.$ & empty \\
\hline $\mathrm{CD}$ & $<0\}$ & ] $1-e^{-\mu_{0}}, 1+e^{-\mu_{0}}[$ \\
\hline
\end{tabular}

We obtained the conditions that a general coupling (4) needs to satisfy in order to admit a complete synchronization or a delayed synchronization. Those conditions led us to consider four types of coupled systems: SL, CL, SD and CD. Both symmetric systems admit a chaotic complete synchronization and a period-2 delayed synchronization (but not a chaotic one). Both commanded systems admit a chaotic synchronization: the CL just admits the complete synchronization; the CD just admits the delayed synchronization. None of the commanded systems admit a period- 2 delayed synchronization. We used an analytical approach to obtain the 
windows of complete synchronization presented in Table 1 . We also used the same analytical approach to obtain the window of chaotic delayed synchronization for the $\mathrm{CD}$, the only considered coupled system that admits a chaotic delayed synchronization. We observed numerically all these windows using different free chaotic dynamics.

As an example of numerical results, we show the zoom in the "Window of complete synchronization" for the SD corresponding to the maps belonging to the family of unimodal maps defined in (1) (on the left) and the maps belonging to the family of bimodal maps defined in (2) (on the right). In these figures, we can see the values of $c$ for which $x_{1}(t)=x_{2}(t)$ within a chaotic trajectory $s(t)$. For more details, see [6].
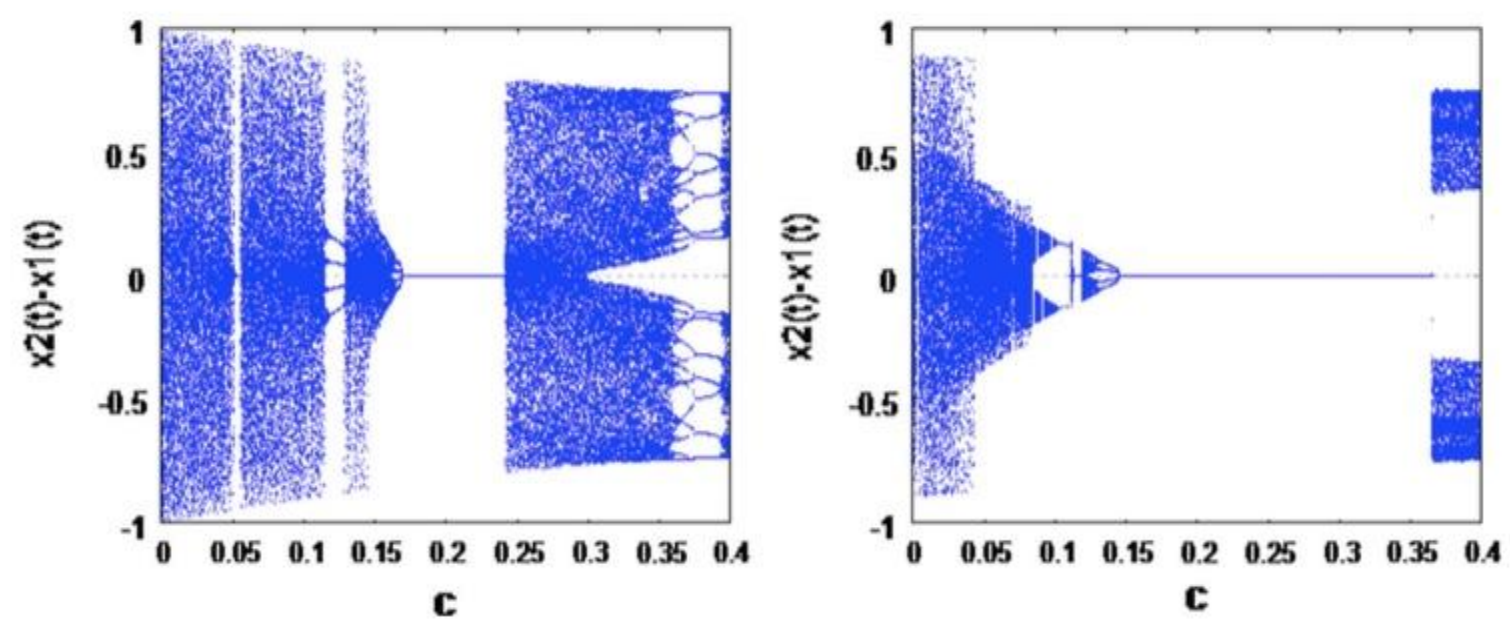

Fig. 5: Zoom in the "Window of complete synchronization" for the SD corresponding to the maps belonging to the family of unimodal maps defined in (1) (on the left) and the maps belonging to the family of bimodal maps defined in (2) (on the right).

\section{Conclusion}

The present paper is just a flavor of two subjects in active research in the field of discrete dynamical systems. With the background of iteration theory, there are very interesting topics as nonautonomous dynamical systems, where the iteration function varies with the time; iteration in $\mathrm{m}$-dimensional systems, where the process lies in an m-dimensional space; complex networks, which are graphs with non- trivial topological features; and many other. The richness of these themes offers young researchers numerous research topics with applications to current topics such as information theory, social networks, psychology, medicine and others.

\section{Acknowledgement}

The first author Sara would like to acknowledge the Erasmus Mundus LEADER Project for its financial support to carry out the work in Pulchowk Campus, Institute of Engineering, Tribhuvan University, Nepal, Oct-Nov, 2017. The third author Gyan would like to acknowledge the Erasmus Mundus LEADER Project 
for its financial support to carry out the work in university of Evora, Portugal as post doc fellow during Nov 2016-Sep 2017.

\section{References}

[1] Almeida P, Lampreia JP and Ramos JS (1996), Topological invariants for bimodal maps, in Iteration Theory (Batschuns, 1992), World Scientific, 1-8.

[2] Buesco J and Ramos JS (1948-2007), (2007), Gazeta da Matemática, nº153.

[3] Fernandes S, Grácio C and Ramos C (2010), Conductance in discrete dynamical systems. Nonlinear Dynamics, 61: 435-442.

[4] Fernandes S, Grácio C and Ramos C (2013), Systoles in discrete dynamical systems, Journal of Geometry and Physics 63: 129-139.

[5] Kitchens BP (1997), Symbolic Dynamics: One-sided, Two-sided and Countable State Markov Shifts (Universitext).

[6] Lopes LM, Fernandes S and Grácio C (2014), Complete synchronization and delayed synchronization in couplings, Nonlinear Dynamics, 76(3), Springer.

[7] Milnor J and Thurston W (1988), On iterated maps of the interval. Dynamical systems (College Park, MD, 1986-87). In: Lecture Notes in Math., 1342, 465-563. Springer, Berlin.

[8] Parry W (1966), Symbolic dynamics and transformations of the unit interval. Trans. Am. Math. Soc. 122: 368-378.

[9] Walters P (2000), An Introduction to Ergodic Theory, in: Graduate Texts in Mathematics, Springer- Verlag, New York. 